\section{Nombres populares y clasificación de las drogas de abuso ilícitas en Chile}

\author{
ANTONIA SATELER ${ }^{1, \mathrm{a}}$, GONZALO PINO ${ }^{1, \mathrm{~b}}$, \\ ALBERTO LÓPEZ ${ }^{1, \mathrm{a}}$, LORENA SILVA ${ }^{1, \mathrm{~b}}$, SANDRA SOLARI $^{1,2,3, \mathrm{~d}}$, \\ BORIS DUFFAU ${ }^{4, b}$, JUAN CARLOS RÍOS ${ }^{1,2,3, b, c}$
}

\section{Popular names and classification of illicit drugs commonly used in Chile}

\begin{abstract}
Background: The consumption of illicit drugs in Chile has increased over the years generating abuse and dependence problems and becoming a public health problem. Aim: To build and disseminate a figure in which health personnel can quickly visualize illicit drugs and their common names, and thus be able to associate them with the effects and risks that are expected in the patient. Material and Methods: A bibliographic review and compilation of information obtained from dealers and drug abusers. Results: A figure and a table were developed. The former illustrates the classification and common names of drugs, while the second indicates the mechanisms of action and effects expected in the body according to each drug of abuse. Conclusions: Illicit drugs have several popular names and various mechanisms of action. When confronting drug consumption this information is crucial to provide an adequate treatment and withdrawal management. However, it is important to keep in mind that this work only represents a management guide and that treatment should always focus on the condition and clinical manifestations of the patient.
\end{abstract}

(Rev Med Chile 2019; 147: 1613-1620)

Key words: Chile; Street Drugs; Substance-Related Disorders.
'Centro de Información

Toxicológica, Facultad de Medicina, Pontificia Universidad Católica de Chile. Santiago, Chile. ${ }^{2}$ Departamento de Laboratorios Clínicos, Facultad de Medicina, Pontificia Universidad Católica de Chile. Santiago, Chile.

${ }^{3}$ Unidad de Farmacología y Toxicología. Facultad de Medicina de la Pontificia Universidad Católica de Chile. Santiago, Chile. ${ }^{4}$ Instituto de Salud Pública. Sección análisis de drogas. Santiago, Chile. aEnfermera/o.

${ }^{b} Q u i ́ m i c o$ farmacéutico. CPhD.

${ }^{\mathrm{d}}$ Médico Cirujano.

Trabajo no recibió financiamiento. Los autores declaran no tener conflictos de interés.

Recibido el 20 de junio de 2019, aceptado el 18 de noviembre de 2019.

Correspondencia a: Dr. Juan Carlos Ríos Centro de Información Toxicológica, Facultad de Medicina, Pontificia Universidad Católica de Chile. Lira 63, Santiago, Chile jriosb@uc.cl

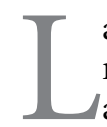

as drogas ilícitas son definidas por la Organización Mundial de la Salud (OMS) como aquellas sustancias cuya "producción, venta y consumo están prohibidos" y que pueden generar cambios en el estado de conciencia, ánimo, procesos de pensamiento y funciones motoras de los individuos que las utilizan. Además, tienen el potencial de generar dependencia ${ }^{1}$. A nivel mundial, el aumento en el consumo de drogas de abuso se ha convertido en un problema de salud pública ${ }^{2}$. Según el último reporte realizado a partir de la encuesta nacional sobre el consumo de drogas del "National Institutes of Health" (NIH) de los Estados Unidos (2018), 49,5\% de las personas de 12 o más años declara haber consumido algún tipo de droga ilegal al menos una vez en su vida, $y$ $19 \%$ reconoce haberlo hecho durante el año $2017^{3}$.
Además de esta gran prevalencia en el consumo, llama la atención que cada vez hay mayor acceso a nuevas drogas de abuso como, por ejemplo, los cannabinoides sintéticos o las nuevas sustancias psicoactivas. Esto se suma a la mayor disponibilidad de drogas, tanto en el caso de la marihuana como de la cocaína.

A nivel nacional, la realidad no está muy alejada de este panorama global, ya que según el $12^{\circ}$ Estudio Nacional de Drogas en Población General del Servicio Nacional para la prevención y rehabilitación del consumo de drogas y alcohol (SENDA) la cantidad de personas que refiere que ha tenido "ofrecimientos" para consumir aumentó en comparación con años anteriores. En el caso de la marihuana, "la proporción que declara haber recibido algún ofrecimiento de 
marihuana durante el último año aumentó significativamente" desde $18,6 \%$ en 2014 a 22,9\% en 2016. Lo mismo ocurre con la cocaína, cuyo ofrecimiento aumentó de 5,2\% el año 2014 a $6,1 \%$ en 2016. El estudio muestra además una tendencia sistemática al alza en el consumo de marihuana en Chile, aumentando la categoría "consumo en el último mes" (personas que han consumido marihuana dentro del último mes) con una prevalencia de $8,7 \%$ en 2016 , significativamente mayor a la prevalencia en estudios anteriores $(6,8 \%$ el año 2014). A lo anterior se suma un aumento desde $31,5 \%$ en 2014 a $37,7 \%$ en 2016 del "consumo de marihuana alguna vez en la vida". El "consumo durante el último año" (medida principal del SENDA para la caracterización del consumo de drogas en Chile) aumenta significativamente alcanzando 14,5\% el año 2016 versus $11,3 \%$ correspondiente al estudio anterior. Todos los tramos de nivel socioeconómico (NSE) presentan alzas en el consumo. Por rango etario, la prevalencia de consumo de marihuana tiende a disminuir levemente en el rango etario de 12 a 18 años, pero aumenta significativamente en los rangos de 19 a 25 y 26 a 34 años, con prevalencias de $33,8 \%$ y $23,4 \%$, respectivamente, lo que genera alzas entre $10 \%$ y $6 \%$ en la prevalencia de cada grupo respecto de la encuesta anterior ${ }^{4}$. En relación al consumo de cocaína, pasta base y otras drogas, presentan una "estabilización" respecto del estudio del año 2014. Solamente la marihuana sintética o spice (incorporada en este grupo para monitoreo por el SENDA), aumenta 1 punto porcentual en su prevalencia, pasando de $1,5 \%$ en 2014 a 2,5\% en 2016. Lo más preocupante de este panorama, es la disminución de la percepción de riesgo frente al "consumo experimental de marihuana (probarla 1 o 2 veces)" de $46,8 \%$ en 2012, $34,4 \%$ en 2014 y $29,2 \%$ en 2016 . La percepción de riesgo del consumo experimental de cocaína desciende no significativamente desde $73,7 \%$ en 2014 a $71,6 \%$ en $2016^{4}$.

De acuerdo a los reportes del Centro de información toxicológica de la Pontificia Universidad Católica de Chile (CITUC), el año 2018, la central de emergencias recibió un universo de 33.647 casos humanos de exposiciones a sustancias potencialmente peligrosas. De ellas, 523 reportes $(1,6 \%)$ corresponden a exposiciones a drogas de abuso. De estos casos, 55,4\% ocurrió con fines de abuso, $22,2 \%$ con intencionalidad suicida y
$14,5 \%$ fueron accidentales. Los individuos expuestos en mayor proporción fueron adultos $(68,1 \%)$, adolescentes $(14,3 \%)$ y menores de 12 años (14,7\%). Al analizar las drogas de abuso de mayor incidencia de reportes se obtuvo que la cocaína junto a la pasta base alcanzan $41,1 \%$, marihuana y creepy (cannabis sativa) $25,5 \%$ y narcóticos-opiáceos (tramadol-oxicodona) 5,8\%. En relación a las drogas de abuso ilícitas en Chile con nombres populares destacan 20 casos vinculados a éxtasis (MDMA), 20 con sospecha de exposición a burundanga (escopolamina), 4 relacionados a droga "tusi" o "cocaína rosa" [2C-B (4-bromo-2,5-dimetoxifeniletilamina)], 3 casos de ketamina, 1 reporte de heroína y 1 caso perteneciente a 25i-nbome (feniletilamina). En $17,6 \%$ de los casos existió co-exposición a bebidas alcohólicas. Si bien, la prevalencia de este tipo de drogas no es significativo desde el punto de vista epidemiológico, si revela que estos agentes están comenzando a ingresar a Chile, por lo que es importante actualizarse respecto al tema. En relación a la sintomatología de los individuos expuestos al momento del llamado a la central de emergencias destacan: taquicardia sinusal $(14,6 \%)$, somnolencia/letargia $(11,8 \%)$, agitación psicomotora $(8,7 \%)$, vómitos $(6,2 \%)$, hipertensión $(4,1 \%)$, midriasis $(3,5 \%)$, dolor precordial $(2,7 \%)$, convulsiones $(2,6 \%)$, sopor $(2,3 \%)$, sudoración $(1,6 \%)$, temblor $(1,5 \%)$ y alucinaciones $(1,3 \%)$, entre otras. Durante el primer semestre de 2019, la central de emergencias de CITUC ha recibido 305 casos, lo que equivale a 58,3\% de los casos reportados durante el 2018.

En Chile, el consumo de sustancias de abuso comienza cada vez a edades más precoces, lo que constituye un factor de riesgo para generar adicción, trastornos mentales e incluso accidentes en los adolescentes. Tomando en cuenta lo anteriormente expuesto, sumado a la capacidad adictiva de muchas de las drogas ilícitas, se puede evidenciar que se genera un impacto considerable en la morbimortalidad de la población, desencadenando además, problemas psicosociales en los consumidores de estas drogas. Esto se traduce en el deterioro en las relaciones interpersonales, intrafamiliares, bajo rendimiento académico y deserción escolar en los estudiantes que presentan consumo de estas drogas 5 . Este consumo y dependencia de drogas de abuso implica distintas consecuencias en la salud física y psíquica de las personas que 
las consumen, y de su entorno más cercano. Se suman a esto las consecuencias derivadas de la forma de administración (como ejemplo, el riesgo de infección en el caso de drogas inyectables) y los problemas agudos por sobredosis, que pueden llegar a ser letales ${ }^{2}$. En este artículo se dará énfasis a la clasificación y efectos tóxicos que estas drogas pueden producir en el contexto del individuo expuesto, entregando orientación para poder identificar las drogas a partir de los nombres de uso popular.

Es importante aclarar y recalcar que este artículo consiste netamente en una guía para orientar al diagnóstico del paciente intoxicado por drogas de abuso ilícitas. Sin embargo, el manejo clínico de este tipo de pacientes siempre debe realizarse a partir del cuadro clínico que estos presenten. En contexto con lo anterior, en reiteradas ocasiones los propios pacientes refieren nombres incorrectos de la droga que consumieron o abiertamente lo desconocen. También es común que, al momento de la compra de estas sustancias, los consumidores no reciban información detallada de la droga, o bien esta puede ser errónea o estar adulterada. Lo anterior lleva a la confusión por parte del equipo de salud, existiendo discordancia evidente entre la sintomatología del paciente y el cuadro clínico esperado para dicha exposición.

\section{Objetivos}

Es por este creciente aumento del consumo de drogas y la disminución de la percepción del riesgo, junto con la aparición de nuevas drogas y mayor facilidad en el acceso, que los profesionales de la salud deben estar actualizados con respecto a esta temática, para así poder brindar respuestas oportunas al verse enfrentados a situaciones de emergencias en las que la salud y la vida del paciente se encuentren en riesgo. A raíz de lo anterior, el objetivo de este artículo es levantar datos por medio de una revisión bibliográfica con el fin de describir, clasificar y señalar los principales nombres de uso común adjudicados a las drogas ilícitas en Chile para representarlos en forma gráfica y detallada a través de un esquema y una tabla. Lo anterior tiene el propósito de caracterizar y actualizar dicha información en un formato que permita al clínico una fácil interpretación, brindando orientación al personal de salud en la identificación de las diversas drogas ilícitas a las que podría haber estado expuesto un paciente in- toxicado, apoyando el diagnóstico. De esta forma, dicho personal tendrá mayor claridad respecto al manejo que requiere cada paciente.

\section{Material y Método}

Para desarrollar este trabajo, se realizó una revisión bibliográfica. En primer lugar, se revisó a través de PubMed con dos términos MeSh: "Street drugs" y "pharmacology" usando el término booleano "AND". De esta búsqueda se obtuvo un total de 268 artículos. Al filtrar los trabajos a aquellos correspondientes a los últimos 10 años, existían 120 artículos disponibles. De estos se seleccionaron 27 que efectivamente permitían cumplir con el objetivo planteado y se descartaron aquellos que incluía abuso de drogas lícitas, como por ejemplo abuso de clonazepam o clorpromazina (excepto la Ketamina y derivados del fentanilo, ya que estas se mezclan con otras sustancias ilícitas y es importante tener esto en consideración).

En segundo lugar, se indagó en la base de datos Micromedex y Poisindex ${ }^{7}$ para recabar en mayor detalle la información de cada droga. Estas bases de datos entregan información muy completa y actualizada sobre distintos agentes tóxicos, sus efectos clínicos, mecanismos de toxicidad y manejo de las intoxicaciones.

Para identificar los nombres informales de drogas de abuso ilícitas entre consumidores y vendedores, se utilizó el buscador Google, focalizando el análisis en páginas como la del SENDA, foros y blogs donde los mismos usuarios se comunican sobre formas de uso e indican nombres coloquiales de estas mismas ${ }^{4}$.

\section{Resultados}

Como resultados de este artículo se desarrolló una figura que muestra la clasificación de las drogas ilícitas según su efecto sobre el sistema nervioso central y los nombres comunes utilizados por consumidores y vendedores. En segundo lugar, se construyó una tabla que apunta a indicar los mecanismos de acción de cada agente y el grado en que se ven afectados los órganos diana.

A continuación, se muestra la clasificación y nombres comunes usados en Chile por los consumidores (Figura 1). En esta figura se muestra una 


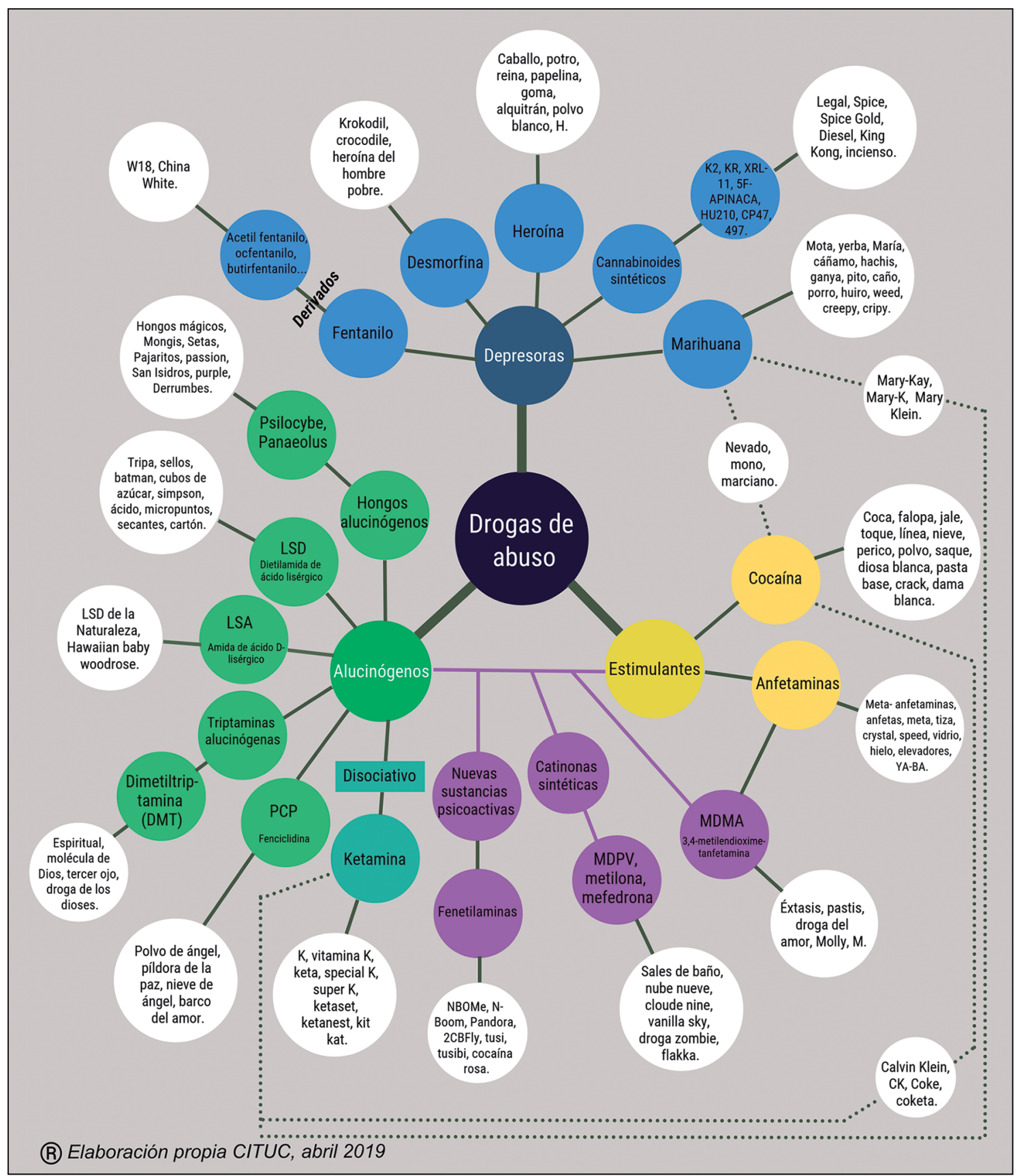

Figura 1. Clasificación de drogas de abuso según su efecto sobre el sistema nervioso central y nombres comunes usados por consumidores y vendedores. Aclaraciones:

En colores se indica el efecto de las drogas sobre el sistema nervioso centra|8,9:

- Azul: drogas depresoras.

- Amarillo: drogas estimulantes.

- Verde: drogas alucinógenas.

- Morado: drogas que tienen tanto efecto alucinógeno como estimulante.

En círculos blancos se señalan los nombres de uso común por consumidores y vendedores.

Las líneas punteadas conectan distintas drogas de abuso para representar mezclas que se realizan. 
clasificación a partir de los efectos que producen estas drogas sobre el sistema nervioso central; depresoras, estimulantes, alucinógenas y estimulantes- alucinógenas. Esta clasificación a grandes rasgos otorga al profesional una aproximación general del cuadro clínico que se puede esperar por la exposición a cada droga. De esta forma, puede prever posibles complicaciones.
Las drogas ilícitas que se tratan en este artículo, producen efectos que pueden afectar distintos órganos o sistemas pero en general, la mayor toxicidad se manifiesta a nivel neurológico y cardíaco ${ }^{7}$.

Dentro de la toxicidad neurológica, los signos y síntomas más comunes consisten en convulsiones, alteraciones de la conducta, memoria y el pensamiento, hiperreflexia y agitación en el caso de las

Tabla 1. Escala de severidad de drogas de abuso

\begin{tabular}{|c|c|c|}
\hline Severidad por sistemas & Droga & Mecanismo de acción \\
\hline+ & Heroína/Fentanilo & $\begin{array}{l}\text { Unión a receptores opiáceos }{ }^{10,11} \\
\text { ** Riesgo de depresión respiratoria }{ }^{12}\end{array}$ \\
\hline & Cannabinoides sintéticos & Estimulación de receptores cannabinoides ${ }^{13-16}$ \\
\hline & Marihuana & Agonista de los receptores cannabinoides $s^{17,18}$ \\
\hline$+\quad$ & Cocaína & $\begin{array}{l}\text { Bloquea re captación de dopamina, noradrenalina y } \\
\text { serotonina }{ }^{19-21}\end{array}$ \\
\hline$+\oplus$ & Anfetaminas & $\begin{array}{l}\text { Aumenta estimulación de receptores adrenérgicos y } \\
\text { liberación de catecolaminas }{ }^{22-26}\end{array}$ \\
\hline$+\oplus$ & MDMA & $\begin{array}{l}\text { Estimulación de la actividad de catecolaminas, dopamina } \\
\text { y serotonina }{ }^{27-30}\end{array}$ \\
\hline+ & Catinonas sintéticas & $\begin{array}{l}\text { Aumenta la liberación de neurotransmisores (dopamina, } \\
\text { serotonina y noradrenalina) }{ }^{31}\end{array}$ \\
\hline$+\oplus$ & $\begin{array}{l}\text { Nuevas sustancias } \\
\text { psicoactivas }\end{array}$ & $\begin{array}{l}\text { Actúan como potentes agonistas del receptor } 2 \mathrm{~A} \\
(5 \mathrm{HT} 2 \mathrm{~A})^{32}\end{array}$ \\
\hline+ & Ketamina & $\begin{array}{l}\text { Antagonista receptor de glutamato no competitivo. En } \\
\text { dosis altas se une a receptores opioides y actúa como } \\
\text { agonista GABA. **Riesgo de depresión respiratoria }{ }^{33-35}\end{array}$ \\
\hline+0 & PCP (Fenciclidina) & $\begin{array}{l}\text { Inhibe recaptura de norepinefrina, epinefrina y } \\
\text { serotonina, provocando estimulación de receptores } \\
\text { adrenérgicos. Además estimula receptores opioides }{ }^{35-37}\end{array}$ \\
\hline+ & $\begin{array}{c}\text { DMT } \\
\text { (dimetiltripta- mina) }\end{array}$ & $\begin{array}{l}\text { Agonista serotoninérgico. Estimulación de receptores } \\
\text { dopaminérgicos y triptamínicos tienen actividad menor } \\
\text { en su acción farmacológica }\end{array}$ \\
\hline 0 & LSD & Agonista serotoninérgico ${ }^{38}$ \\
\hline$\oplus \quad+$ & Hongos alucinógenos & Agonista serotoninérgico 39 \\
\hline
\end{tabular}

Cada sistema se presenta por medio de un círculo de un color en específico: Neurotoxicidad; C Cardiotoxicidad; $\bigcirc$ Nefrotoxicidad; - Hepatotoxicidad. Además de los colores, los círculos tienen distintos tamaños y contienen cruces que representan el grado de severidad que tiene la droga en cada órgano y/o sistema. A mayor tamaño y número de cruces, mayor toxicidad en cada sistema. 
drogas psicoestimulantes. En aquellas con efecto depresor sobre el sistema nervioso, se espera somnolencia y letargia. En el caso de la cocaína también se espera como síntoma neurológico importante, el riesgo de hemorragia intracraneal, infarto cerebelar y coreoatetosis. Los efectos de cadiotoxicidad que se manifiestan en estos pacientes se relacionan principalmente a alteraciones en el ritmo cardiaco y de la presión arterial. Adicionalmente podrían existir alteraciones de la irrigación, provocando infarto al miocardio. Otras manifestaciones clínicas asociadas al consumo de drogas de abuso, corresponden al riesgo de nefrotoxicidad (retención urinaria y falla renal) y a hepatotoxicidad (alteración de enzimas hepáticas y falla hepática) ${ }^{7}$.

Todos estos signos y síntomas descritos en la literatura se correlacionan con los síntomas referidos en los reportes de pacientes que ingresaron al CITUC.

Resulta fundamental conocer el mecanismo de acción de cada una de las drogas ilícitas al momento de enfrentar los casos de consumo, lo que permite anticipar sus consecuencias. Para tal efecto y según la información recopilada, se construyó una tabla con la toxicodinamia de cada agente, en la cual se señala el grado de toxicidad por cada órgano y sistema (Tabla 1).

Se debe recordar que a pesar de que los efectos agudos en caso de intoxicación pueden ser graves e incluso letales, pueden existir otras manifestaciones clínicas ante exposiciones crónicas, como es el caso de infecciones por consumo de drogas inyectables, o lesiones producto del consumo de cocaína. Una complicación frecuente es la aparición de pseudovasculitis, la cual consiste en la "destrucción de estructuras óseas y cartilaginosas nasales y sinusales", lo anterior se produce por la vasoconstricción e isquemia local que genera el consumo de cocaína ${ }^{40}$.

La prevalencia de consumo en la población es alta, lo que representa un problema de salud pública $^{41}$. En esto último radica la importancia, para los profesionales de la salud, de actualizarse constantemente, respecto de esta temática. A pesar de describir los nombres de uso común de los distintos agentes y señalar los efectos que se esperan tras su consumo, se recalca la importancia de tratar siempre al paciente según su sintomatología y cuadro clínico, ya que es posible que no haya total seguridad del agente consumido por el paciente.

\section{Conclusiones}

Frente a todo lo expuesto se puede concluir que las drogas antes señaladas presentan variabilidad en cuanto a su mecanismo de acción, algunos incluso son antagónicos. Al mismo tiempo, existe diversidad de nombres utilizados por los consumidores para un mismo agente, y esto no tiene relación con el mecanismo de acción de cada droga. Estos nombres no siguen un patrón específico en su denominación.

Este artículo representa una guía para el diagnóstico de intoxicaciones por drogas de abuso ilícitas, sin embargo, el tratamiento debe ser adecuado según los antecedentes de cada caso, las circunstancias de exposición y las condiciones del paciente.

\section{Referencias}

1. OMS. Management of substance abuse [Internet]. Organización Mundial de la Salud; 1994 [Consultado el 19 de marzo de 2019]. Disponible en: https://www.who.int/ substance_abuse/terminology/who_ladt/en/

2. Observatorio Europeo de las Drogas y las Toxicomanías. Informe Europeo sobre Drogas: Tendencias y novedades [Internet]. Luxemburgo: Oficina de Publicaciones de la Unión Europea; 2018 [Consultado el 19 de marzo de 2019]. Disponible en: http://www.emcdda.europa. eu/system/files/publications/11364/20191724_TDAT19001ESN_PDF.pdf

3. Center for Behavioral Health Statistics and Quality. 2017 National Survey on Drug Use and Health: Detailed Tables [Internet]. Rockville, MD: Substance Abuse and Mental Health Services Administration; 2017 [Consultado el 20 de marzo de 2019]. Disponible en: https:// www.samhsa.gov/data/sites/default/files/cbhsq-reports/ NSDUHDetailedTabs2017/NSDUHDetailedTabs2017. pdf.

4. SENDA. Décimo Estudio Nacional de Drogas en Población General de Chile [Internet]. Chile: Observatorio Chileno de drogas; 2017 [Consultado el 20 de marzo de 2019]. Disponible en: http://www.senda.gob.cl/wp-content/uploads/2017/12/InformeENPG2016.pdf.

5. Gaete J, Olivares E, Rojas-Barahona CA, Rengifo MJ, Labbé N, Lepe L, et al. Consumo de tabaco y alcohol en adolescentes de 10 a 14 años de la ciudad de San Felipe, Chile: prevalencia y factores asociados. Rev Med Chile 2016; 144 (4): 467-75. Disponible en: www.scielo.cl [Consultado el 21 de mayo de 2019]. 
6. Roberts E, Gooch MD. Pharmacologic strategies for treatment of poisoning. Nurs Clin North Am 2016: 51 (1): 57-68. Disponible en https://www.ncbi.nlm.nih.gov/ pubmed/26897424 [Consultado el 25 de abril de 2019].

7. IBM Micromedex ${ }^{\circledR}$ POISINDEX $^{\circledR}$ : POISINDEX ${ }^{\circledR}$ System [Internet]. Greenwood Village, CO: Truven Health Analytics. c2019 - [Consultado el 20 de abril de 2019]. Disponible en http://www.micromedexsolutions.com

8. Essel K, Fossati B, Traylor A, Martin-Schild S. Epidemic of illicit drug use, mechanisms of action/addiction and stroke as a health hazard. Brain behav 2011; 1 (1): 44-54. Disponible en: www.ncbi.nlm.nih.gov [Consultado el 21 de abril de 2019].

9. Ciccarone D. Stimulant abuse: pharmacology, cocaine, methamphetamine, treatment, attempts at pharmacotherapy. Prim Care 2011; 38 (1): 41-58. Disponible en: www.ncbi.nlm.nih.gov [Consultado el 21 de abril de 2019].

10. Bolin BL, Alcorn JL, Reynolds AR, Lile JA, Stoops WW, Rush CR. (2016). Human Drug Discrimination: Elucidating the Neuropharmacology of Commonly Abused Illicit Drugs. Curr Top in Behav Neurosci 2018; 39: 261295. Disponible en: www.ncbi.nlm.nih.gov [Consultado el 21 de abril de 2019].

11. Griffiths B, Willms L, Jayathissa S. AV conduction block and proximal myopathy induced by Gee's cough linctus. N Z Med J 2009; 122 (1293): 67-9. Disponible en: www. ncbi.nlm.nih.gov [Consultado el 20 de febrero de 2019].

12. Jolley CJ, Bell J, Rafferty GF, Moxham J, Strang J. Understanding heroin overdose: a study of the acute respiratory depressant effects of injected pharmaceutical heroin. PLoS One 2015; 10 (10): 1-14. Disponible en: www.ncbi.nlm.nih.gov [Consultado el 20 de febrero de 2019].

13. Buser GL, Gerona RR, Horowitz BZ, Vian KP, Troxell ML, Hendrickson RG, et al. Acute kidney injury associated with smoking synthetic cannabinoid. Clin Toxicol (Phila) 2014; 52 (7): 664-73. Disponible en: www.ncbi. nlm.nih.gov [Consultado el 20 de febrero de 2019].

14. Schneir AB, Baumbacher T. Convulsions associated with the use of a synthetic cannabinoid product. J Med Toxicol 2012; 8 (1): 62-4. Disponible en: www.ncbi.nlm. nih.gov [Consultado el 20 de febrero de 2019].

15. Andonian DO, Seaman SR, Josephson EB. Profound hypotension and bradycardia in the setting of synthetic cannabinoid intoxication - a case series. Am J Emerg Med 2017; 35 (6): 940. Disponible en: www.ncbi.nlm. nih.gov [Consultado el 20 de febrero de 2019].

16. Mir A, Obafemi A, Young A, Kane C. Myocardial infarction associated with use of the synthetic cannabinoid K2. Pediatrics 2011; 128 (6): 1622-7. Disponible en: www.ncbi.nlm.nih.gov [Consultado el 21 de febrero de 2019].

17. Fitzerald K, Bronstein A, Newquist K. Marijuana Poisoning. Topics in compan an med 2013; 28 (1): 8- 12. Disponible en: www.ncbi.nlm.nih.gov [Consultado el 21 de febrero de 2019].

18. Rezkalla S, Kloner RA. Cardiovascular effects of marijuana. Trends Cardiovasc Med 2018. Disponible en: www.ncbi.nlm.nih.gov [Consultado el 21 de febrero de 2019].

19. Irani F, Kasmani R, Kanjwal Y. Hyperkalemia and cocaine induced dynamic Brugadatype electrocardiogram. Eur J Emerg Med 2009; 17 (2): 113- 5. Disponible en: www.ncbi.nlm.nih.gov [Consultado el 21 de febrero de 2019].

20. Armenian P, Fleurat M, Mittendorf G, Olson KR. Unintentional pediatric cocaine exposures result in worse outcomes than other unintentional pediatric poisonings. J Emerg Med 2017; 52 (6): 825- 32. Disponible en: www. ncbi.nlm.nih.gov [Consultado el 21 de febrero de 2019].

21. Guollo F, Narciso-Schiavon JL, Barotto AM, Zannin M, Schiavon LL. Significance of alanine aminotransferase levels in patients admitted for cocaine intoxication. J Clin Gastroenterol 2015; 49 (3): 250- 5. Disponible en: www.ncbi.nlm.nih.gov [Consultado el 21 de febrero de 2019].

22. Steidl KE, Darko W, Probst LA, Noviasky JA, Nasser, S. Rhabdomyolysis associated with phentermine. Am J Health Syst Pharm 2010; 67 (22): 1929-32. Disponible en: www.ncbi.nlm.nih.gov [Consultado el 23 de febrero de 2019].

23. Kaland ME, Klein-Schwartz W. Comparison of lisdexamfetamine and dextroamphetamine exposures reported to U.S. poison centers. Clin Toxicol 2015; 53 (5): 477-85. Disponible en: www.ncbi.nlm.nih.gov [Consultado el 23 de febrero de 2019].

24. Mladěnka P, Applová L, Patočka J, Costa VM, Remiao F, Pourová J et al. Comprehensive review of cardiovascular toxicity of drugs and related agents. Med Res Rev 2018; 38 (4): 1332-403. Disponible en: www.ncbi.nlm.nih.gov [Consultado el 21 de abril de 2019].

25. Richards JR, Lange RA, Arnold TC, Horowitz BZ. Dual cocaine and methamphetamine cardiovascular toxicity: rapid resolution with labetalol. Am J Emerg Med 2017; 35 (3): 519.e1-519e.4. Disponible en: www.ncbi.nlm.nih. gov [Consultado el 21 de abril de 2019].

26. Halpin L, Gunning W, Yamamoto B. Methamphetamine causes acute hyperthermia-dependent liver damage. Pharmacol Res Perspect 2013; 1 (1): 1-11. Disponible en: www.ncbi.nlm.nih.gov [Consultado el 21 de abril de 2019]. 
27. Davies O, Batajoo-Shrestha B, Sosa-Popoteur J, Olibrice $\mathrm{M}$. Full recovery after severe serotonin syndrome, severe rhabdomyolysis, multi-organ failure and disseminated intravascular coagulopathy from MDMA. Heart Lung 2014; 43 (2): 117-9. Disponible en: www.ncbi.nlm.nih. gov [Consultado el 24 de febrero de 2019].

28. Ginat DT. MRI of toxic leukoencephalopathy syndrome associated with methylenedioxymethamphetamine. Neurology 2015; 84 (7): 757. Disponible en: www.ncbi. nlm.nih.gov [Consultado el 24 de febrero de 2019].

29. Hoggett K, McCoubrie D, Fatovich DM. Ecstasy-induced acute coronary syndrome: something to rave about. Emerg Med Australas 2012; 24 (3): 339-42. Disponible en: www.ncbi.nlm.nih.gov [Consultado el 24 de febrero de 2019].

30. Colak Y, Tuncer I, Enc FY, Ozturk O, Kiziltas S, Ulasoglu C. Ecstasy induced fatal hepatic failure. J Gastrointestin Liver Dis 2011; 20 (2): 215- 6. Disponible en: www.ncbi.nlm.nih.gov [Consultado el 24 de febrero de 2019].

31. Imam S, Patel H, Mahmoud M, Prakash N, King M, Fremont R. Bath Salts Intoxication: A Case Series. J Emerg Med 2013; 45 (3): 361-5. Disponible en: www.ncbi.nlm. nih.gov [Consultado el 24 de febrero de 2019].

32. Dean BV, Stellpflug SJ, Burnett AM, Engebretsen KM. 2C or not 2C: phenethylamine designer drug review. J Med Toxicol 2013; 9 (2): 172-8. Disponible en: www. ncbi.nlm.nih.gov [Consultado el 24 de febrero de 2019].

33. Yiu-Cheung C. Acute and Chronic Toxicity Pattern in Ketamine Abusers in Hong Kong. J Med Toxicol 2012; 8 (3): 267-70. Disponible en: www.ncbi.nlm.nih.gov [Consultado el 26 de febrero de 2019].

34. Wang JW, Kivovich V, Gordon L. Ketamine abuse syndrome: hepatobiliary and urinary pathology among adolescents in Flushing, NY. Pediatr Emerg Care 2017; 33 (8): 24-6. Disponible en: www.ncbi.nlm.nih.gov
[Consultado el 26 de febrero de 2019].

35. Barton $\mathrm{CH}$, Sterling ML, Vaziri ND. Rhabdomyolysis and acute renal failure associated with phencyclidine intoxication. Arch Intern Med 1980; 140 (4): 568-569. Disponible en: www.ncbi.nlm.nih.gov [Consultado el 26 de febrero de 2019].

36. Carroll F, Lewin A, Mascarella S, Seltzman H, Reddy P. Designer drugs: a medicinal chemistry perspective. Annals of the New York Academy of Sciences: 2011; 1248 (1): 18-38. Disponible en: www.ncbi.nlm.nih.gov [Consultado el 26 de abril de 2019].

37. Zidkova M, Hlozek T, Balik M, Kopecky O, Tesinsky P, Svanda J, et al. Two cases of non-fatal intoxication with a novel street hallucinogen: 3-methoxy-phencyclidine. J Anal Toxicol 2017; 41 (4): 350- 4. Disponible en: www. ncbi.nlm.nih.gov [Consultado el 4 de marzo de 2019].

38. Berrens Z, Lammers J, White C. Rhabdomyolysis After LSD Ingestion. Psychosomatics 2010; 51 (4): 356. Disponible en: www.ncbi.nlm.nih.gov [Consultado el 4 de marzo de 2019].

39. White J, Weinstein SA, De Haro L, Bédry R, Schaper A, Rumack BH, et al. Mushroom poisoning: A proposed new clinical classification. Toxicon 2019; 157: 53- 65. Disponible en: www.ncbi.nlm.nih.gov [Consultado el 4 de marzo de 2019].

40. Barrera M, De la Rivera M, Vela J, Barrera R, Mönckeberg G. Lesiones destructivas de la línea media facial secundarias al consumo de cocaína. Caso clínico. Rev Med Chile 2018; 146 (9): 1070-3. Disponible en: www. scielo.cl [Consultado el 21 de mayo de 2019].

41. Vilugrón F, Hidalgo-Rasmussen CA, Molina T, Gras ME, Font-Mayolas S. Uso de sustancias psicoactivas y calidad de vida relacionada con la salud en adolescentes escolarizados. Rev Med Chile 2017; 145 (12): 1525-34. Disponible en www.scielo.cl [Consultado el 21 de mayo de 2019]. 